\title{
Long-term Preservation of Acousmatic Works: Toward a Generic Model of Description
}

\author{
N. Esposito ${ }^{1}$ and Y. Geslin ${ }^{2}$
}

\begin{abstract}
Acousmatic works are defined at INA-GRM as pure recorded music that is without live instrument or electronic interaction. Usually, the archive of those pieces consist on a single final tape and its security copies, nowadays a single digital file. In the framework of the CASPAR project ${ }^{3}$, it appears to us that it is very important to archive more elements (variants, source elements, notes, etc.) to perform intelligibility preservation over the long-term. Our proposal is to archive these elements regarding the life cycle of an acousmatic work. We provide a model to represent such a life cycle. This model is based on a method which starts with files to finally get a description of the life cycle. This method was applied to a well-documented work: the Hans Tutschku's recent piece Distance Liquide. A model was built on the description of this work. It was validated with other acousmatic works. Then, it can be considered as a generic acousmatic model. It currently uses the CIDOC-CRM ontology.
\end{abstract}

Index Terms-Art, Design methodology, Knowledge representation, Modeling, Long-term preservation, Acousmatic music.

\section{INTRODUCTION}

Acousmatic works are defined at INA/GRM as pure recorded (tape) music that is without live instrument or electronic interaction. Usually, the archive of those pieces consist on a single final tape and its security copies, nowadays a single digital file.

In the framework of the CASPAR project [2], we focus on long-term preservation. And then, we have to preserve the intelligibility of a work over the long-term. Thus, it appears to us that it is very important to archive more elements of an acousmatic piece, i.e. the final mixing session and their variant, and furthermore the source elements. This is proposed mainly to ensure the possibility of enhancing the sound quality in further times.

It means that we need a model to describe this kind of work, to architecture this information into an archive. This paper proposes such a model. We will present our methodology (starting from the files we collect) and the generic model we obtained which is based on a life cycle representation.

\footnotetext{
${ }^{1}$ Nicolas Esposito, CNRS UMR Heudiasyc, Centre de recherches, 60200 Compiègne, France

${ }^{2}$ Yann Geslin, INA/GRM, Maison de Radio-France, 75220 Paris cedex 16, France

3 CASPAR project: http://www.casparpreserves.eu/ STFC (CCLRC) David L. Giaretta, project leader, ACS, Asemantics, Ciant, Engineering, ESA, Forth, IBM Haifa, Ina, Ircam, Metaware, Unesco, Univ. Glasgow, Univ. Leeds, Univ. Urbino.
}

\section{Methodology}

The model to represent acousmatic works was built by applying a method on several acousmatic works. This method is described below. The first work was Hans Tutschku's recent piece Distance Liquide, because we had numerous files about it. It provided us a first model that we confirmed and validated with other acousmatic works.

We use a file-based method to describe an acousmatic archive: the files give us a first group of elements that we can link. These files have to be collected, selected, and classified. Then, they can be used as a first version of each level of information (e.g. main creation flow, additional information about the main creation flow, documents and rights). This first version can be completed later and we can also look for new files.

The files about our examples were collected by Yann Geslin, researcher but also specialist and composer of electroacoustic music, with help of the composers. This was made either after the creation process, either during the process in the case of Hans Tutschku's work. Collecting these files during the creation process allows to avoid missing documents and to create documents especially for an archive purpose (for example: videos of a rehearsal). These files can be collected by an archivist, by a musical assistant, or by the composer himself. It depends on the organization of the institution.

\section{A. Main Steps}

Collecting files - All files directly related to the work (e.g. work items, files about the work). Files were collected from the GRM hard drives and from the composers themselves, and some files were produced especially for the archive (e.g. photos and videos). See figure 1.

Selecting files-Keeping only pertinent files (e.g. removing duplicates). Some files and folders were not selected. For example, a low resolution photo because a high resolution of this photo was in another folder. Another example, a folder was not selected because it was outdated (another folder contained the same files, but newer).

Defining levels of information and classifying filesChoosing, for each file, a level of information. We defined four levels of information: main creation flow, additional information about the creation flow, documents, and rights. And then, we classified the selected files by using these four levels. These levels of information will be described later in this paper.

Modeling the main creation flow-The answer to the questions "who did what?" from the author to the performance.

Adding more information-Alternative versions, equipment, etc. 
Adding documents-Photos, diagrams, reviews, etc. Adding rights - Moral rights, commercial rights, etc.

More steps could be added, for example to deal with authenticity: information that could be used to validate the authentic status of an element.

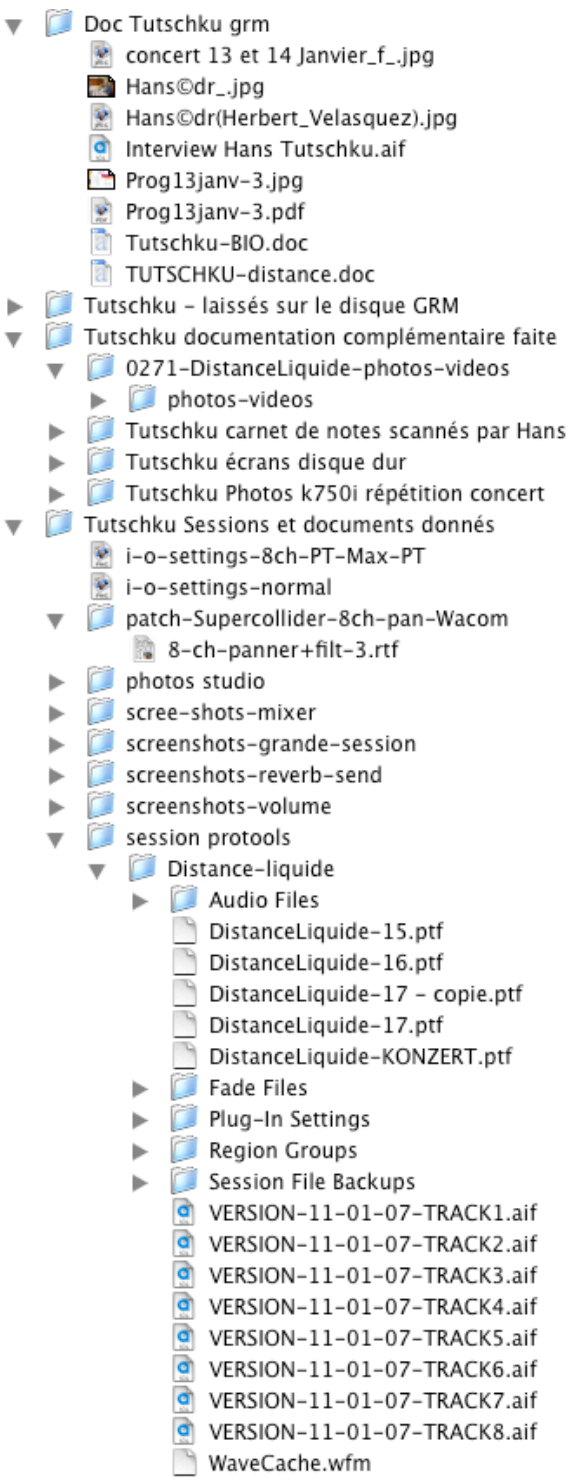

Fig. 1. Collected files for Distance Liquide.

\section{A. Distance Liquide}

Our model is based on a study of the Hans Tutschku's recent piece Distance Liquide. This is electroacoustic music, more precisely: an acousmatic work that uses eight tracks. Its length is 13 minutes. Distance Liquide was commissioned by the GRM (Groupe de Recherches Musicales: Group of Musical Research). The first concert was in January 2007.

\section{B. Graphic Representation}

The CIDOC-CRM ontology [1] is used to describe the life cycle of Distance Liquide and our model. This ontology is an ISO standard and it is widely used in the CASPAR project. We have XML files, but we can also draw graphic representations of these life cycles (see figure 2). Note: each element of the life cycle can be here for one file or more.

Everything is not present on this kind of graphic representation, but it is useful to have the main structure. Especially, rights are not shown. And the compact disc publishing is not included to keep the diagram on one page.

\section{GENERIC MODEL}

The following model (see figure 3) comes from the representation of Distance Liquide. It is generic enough for our other examples: C. Zanési's Aube Rouge, D. Teruggi's Spaces of Mind, and J.Cl. Risset's Sud. It does not mean that it is $100 \%$ generic. But we will see that this is a flexible model. It can easily be adapted to more complete archives (or simpler archives) and future kinds of acousmatic works (see section IV).

\section{A. Levels of Information}

We defined four levels of information: main creation flow (first column of the diagram), additional information about the creation flow (second column), documents (third column), and rights. The fourth one is not present on the graphic representation (it would be to big). These levels of information are useful to categorize the elements of the lifecycle.

Main Creation Flow-This represents the creation flow from the author to the performance. It answers to this question: Who did what?

Additional Information About the Creation Flow-This level focuses on two main kinds of information: alternative versions and details about the elements of the main creation flow.

Documents-Documents that document the elements of the other levels of information.

Rights-Rights that protect the other items. You will find just below details about the expression of the rights.

\section{B. Model Description}

Here is an abstract of the model in English:

- a composer, which is documented by a biography, a photo, and a website, creates sounds,

- these sounds are used to create sequencer files which are documented by screenshots,

○ preliminary sequencer files are also created,

0 the creation of these files is documented by notes and photos,

- these sequencer files are used to create a multitrack master which is documented by an interview and a program note,

O a stereo rendering is also created,

$\circ$ the creation of these masters is commissioned by a commissioner,

- the multitrack master is used to create a spatial projection, using a sound setup which is documented by a setup diagram, and during a concert which is documented by a concert program,

o a rehearsal, which is documented by photos and videos, is also created,

- the photos of the creation of the sequencer files contain the photo of the composer, 


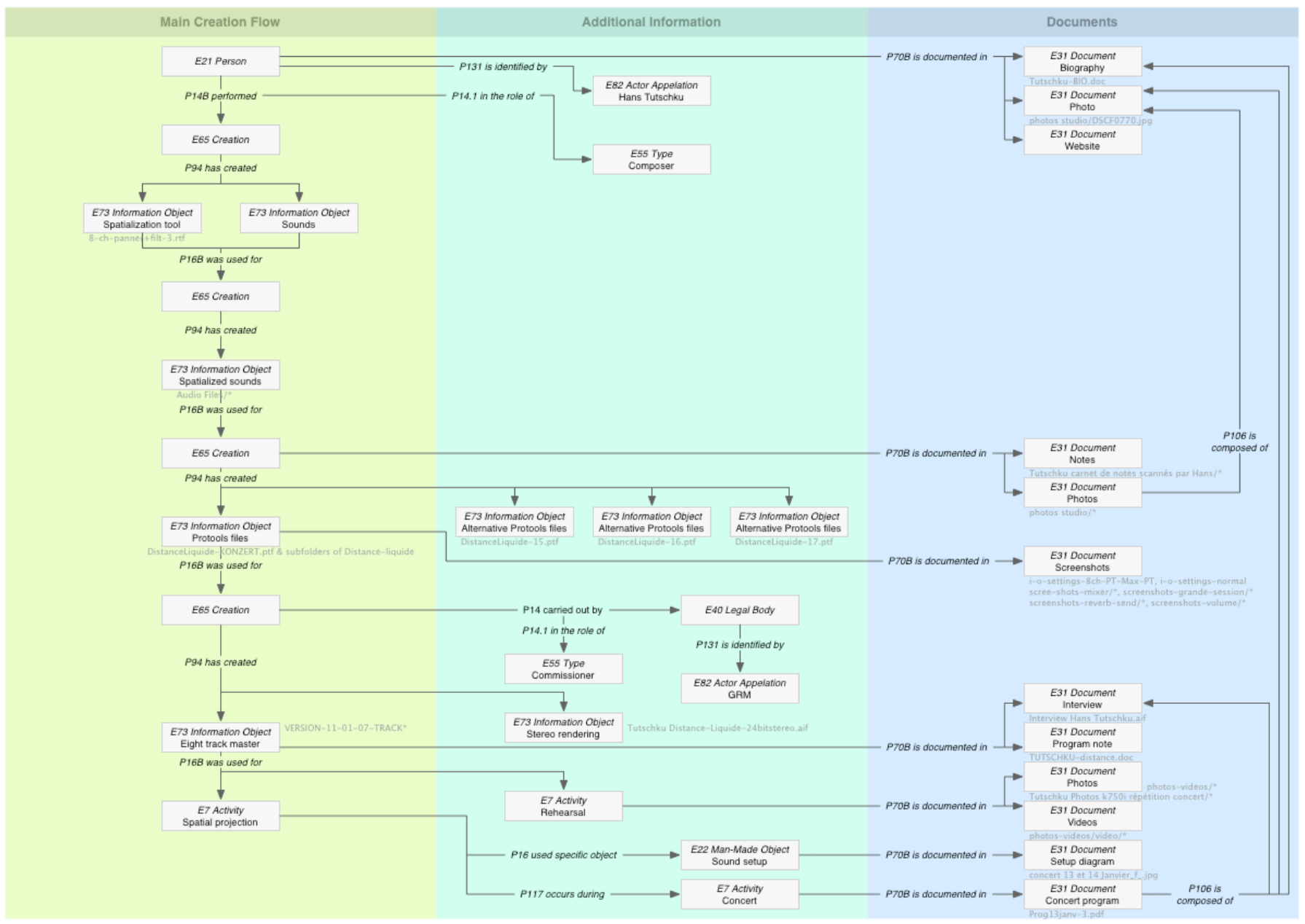

Fig. 2. Graphic representation of Distance Liquide (italic: CIDOC CRM classes and properties; grey: filenames).

\section{Rights}

The rights are not present on the diagram of the model because the image would be too big. Here is a way to express them combining CIDOC-CRM and another ontology. CIDOCCRM can be used to say: An object is subject to a right which is possessed by somebody. And another ontology can be used to describe this right.

We deal with: persons (i.e. Hans Tutschku), organizations (i.e. GRM), groups of people (i.e. musicologists), objects (i.e. an eight track master), and rights (i.e. moral rights).

We also need relations: an object is subject to a right, a right is possessed by someone.

CIDOC-CRM allows us to describe this kind of information. Here is an example:

- the eight track master is subject to moral rights (E73 Information Object P104 is subject to E30 Right),

- the moral rights are possessed by Hans Tutschku (E30 Right P75 is possessed by E21 Person),
- the eight track master is subject to economic rights (E73 Information Object P104 is subject to E30 Right),

- the economic rights are possessed by the GRM (E30 Right P75 is possessed by E40 Legal body).

To have more details about rights, we need to use another ontology. Here are the different types of rights that we have to manage and an example of who is involved:

- privacy: a person on a document (i.e. Hans Tutschku on a photo),

- moral: a person (i.e. Hans Tutschku),

- economic: an organization (i.e. GRM),

- reproduction: an organization (i.e. GRM),

- studying: a group of people (i.e. musicologists),

- reading: a group of people (i.e. everybody),

- browsing: a group of people (i.e. everybody).

We also need to provide details about the rights, i.e. to describes the length of a right and how this right can be transmit. 


\section{Not Showed on the Graphic Representation}

We saw that everything is not showed on the graphic representation. Here some more elements which are not showed, but which are included in the model.

- when the creator involved in a creation is the same for the next creations, it is showed only one time,

- if an element can be considered as the work (i.e. the multitrack master), a type can be added (in CIDOCCRM: P101 had as general use + E55 Type),

- we can see on the graphic representation of the model that we have the preliminary files (in the additional information column) next to the sequencer files (in the main creation flow column) and we can specify the kind of version for each set of file (in CIDOC-CRM: P101 had as general use + E55 Type).

Here are the kinds of versions we need:

- preliminary version (before the creation version),

- creation version (that was ordered),

- next version (after the creation version),

- new release version (which cancels the previous ones),

- declension version (which uses a different format).

\section{EVALUATION AND DiscUSSION}

The model is based on the Distance Liquide description because it is a well-documented work. The life cycles of the other GRM acousmatic works are close to the Distance Liquide one.

Thus, the model was tested by describing three other works (Aube Rouge, Spaces of Mind, and Sud). The result is that the model was used without change.

Moreover, to be compatible with older works, future works, and very specific works, the model comes with special instructions about its possible adaptations.

Optional Items - This model contains a large collection of documents. These documents are good examples of optional items. If an archive producer does not have a particular document, then his representation becomes smaller than the model. It is also true for the other kinds of items (i.e. the stereo rendering or the preliminary files).

Specific Parts-When we look closer at the Distance Liquide representation, we can see that the creation of the spatialized sounds is specific. We do not just have sounds before the sequencer files, we have sounds which are spatialized by using a spatialization tool. This kind of specific part does not need to modify the main structure of the model. It is only a more detailed part of the representation. This can

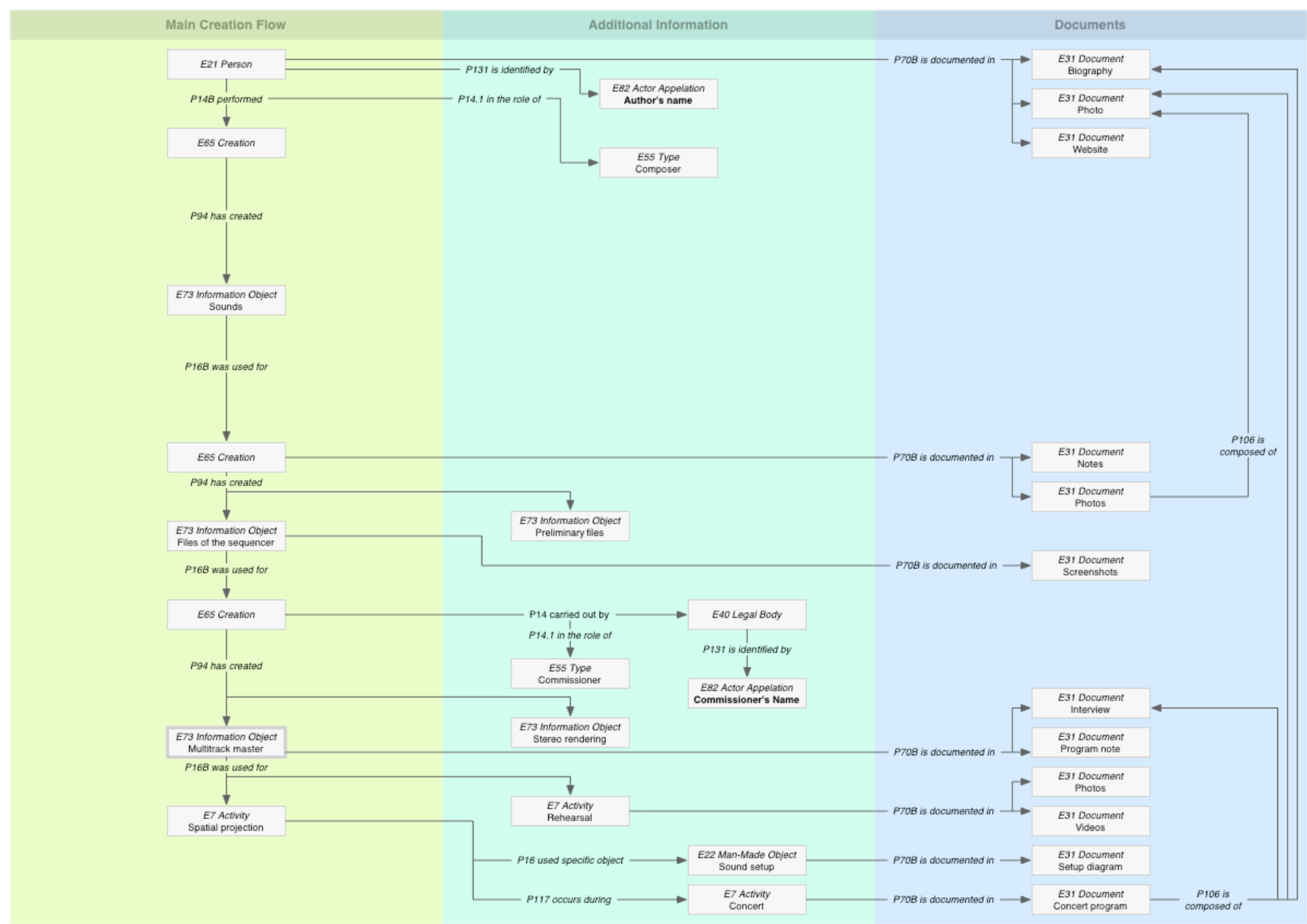

Fig. 3. Graphic representation of the acousmatic model (italic: CIDOC CRM classes and properties). 
be done about each item of the model.

Repetitions-The Distance Liquide representation contains three preliminary sets of files (alternative Protools mixing session files). It shows how we can repeat an item of the model. We could also have this kind of repetition if we would have more than one alternative rendering of the multitrack master (i.e. a 5.1 rendering).

\section{CONCLUSION AND FUtURE WORK}

We provide a model to represent an acousmatic work. This model is based on a method to describe the lifecycle of this kind of work. This method starts with files to finally get a representation of the lifecycle. This method was applied to a well-documented work: the Hans Tutschku's recent piece Distance Liquide. A model was built on the representation of this work. This model was confirmed with other acousmatic works.
Then, it can be considered as a generic acousmatic model, in particular because it comes with special instructions about its possible adaptations. This model currently uses the CIDOCCRM ontology and needs another language to describe the details of the rights.

Now, we are working on the user interface for the archive producer. The first step was to define an acousmatic vocabulary. Indeed, an archive producer is not supposed to handle CIDOC-CRM classes and properties. So, our acousmatic vocabulary was mapped to the CIDOC-CRM ontology. Then, the vocabulary is simple for the archive producer, and the life cycle structure is simplified. Our current activity is to develop a prototype of the user interface and a template to accelerate the archive production (see figure 4). The next steps will be to focus on the authenticity of the archived elements and to study how to connect our approach with the CASPAR components.

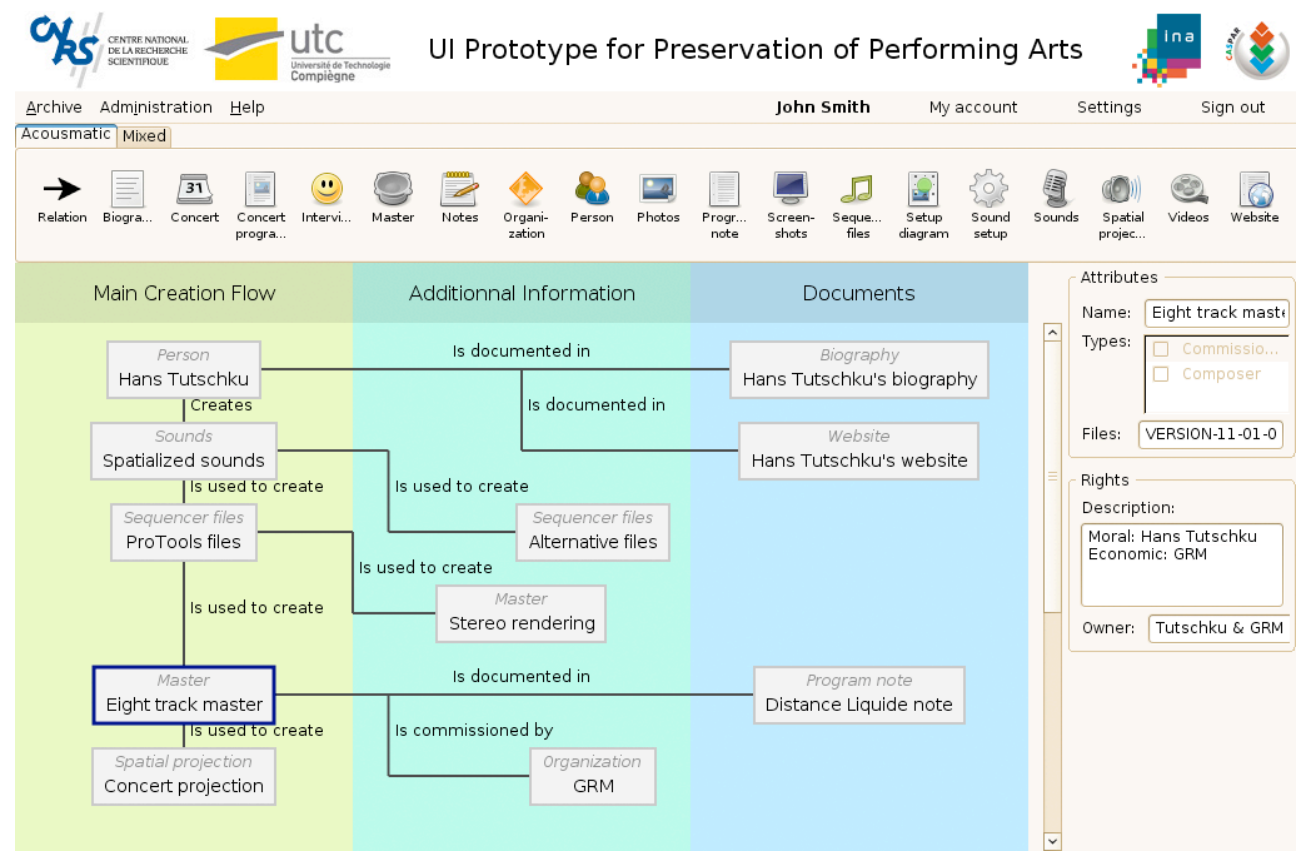

Fig. 4. Prototype of a user interface for an archive producer.

\section{REFERENCES}

[1] Doerr, M. (2003). The CIDOC-CRM - An Ontological Approach to Semantic Interoperability of Metadata. AI Magazine, 24(3). Available: http://cidoc.ics.forth.gr/docs/ontological_approach.pdf

[2] Giaretta et al. (2006). CASPAR and a European Infrastructure for Digital Preservation. European Research Consortium for Informatics and Mathematics, News 66. Available:

http://www.ercim.org/publication/Ercim_News/enw66/giaretta.html

\section{BIOGRAPHIES}

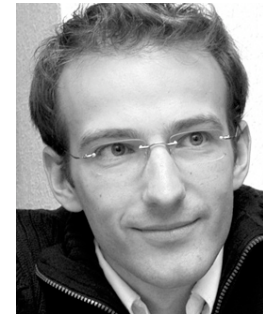

Nicolas Esposito was born in France (1975). He received his $\mathrm{Ph} . \mathrm{D}$. degree in computer science from the National Polytechnic Institute of Grenoble (France, 2002) and his engineering degree in computer science from the University of Technology of Compiègne (France, 1998).

His current main activities are research (artistic work preservation in the CASPAR project at CNRS in Compiègne) and teaching (digital contents at the University of Technology of Compiègne).

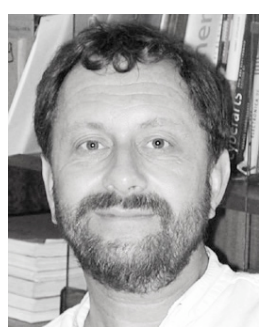

Yann Geslin was born near Vichy in France (1953). $\mathrm{He}$ is currently a Research Project Manager of the "Groupe de Recherches Musicales", part of the Research and Experimentation Department of INA.

He has been associated with the development of the GRM's sound transformation tools since 1977, on mini computers and later on personal systems: the 123 Software, the Syter System and the GRM Tools. He has more recently worked on sound description, representations and information retrieval researches: the Acousmographe 3 software (2005-2006), the CASPAR European IST Project (2006-2008), the Mustica (2004-2005) and Écrins (2002) projects.

$\mathrm{He}$ has studied classical music at the French "Conservatoire National Supérieur de Musique de Paris", and since 1987, he has been teaching a "New Technologies applied to Composition Cursus" there. He has written scientific and dictionary articles and has played and composed computer and electroacoustic music. 